\title{
A Graph-Based Framework for Thermal Faceprint Characterization
}

\author{
Daniel Osaku ${ }^{1, \star}$, Aparecido Nilceu Marana ${ }^{1}$, and João Paulo Papa ${ }^{2, \star \star}$ \\ Department of Computing, São Paulo State University - UnEsP, Bauru, Brazil \\ dosaku@uol.com.br, \{nilceu,papa\}@fc.unesp.br
}

\begin{abstract}
Thermal faceprint has been paramount in the last years. Since we can handle with face recognition using images acquired in the infrared spectrum, an unique individual's signature can be obtained through the blood vessels network of the face. In this work, we propose a novel framework for thermal faceprint extraction using a collection of graph-based techniques, which were never used to this task up to date. A robust method of thermal face segmentation is also presented. The experiments, which were conducted over the UND Collection $\mathrm{C}$ dataset, have showed promising results.
\end{abstract}

Keywords: Faceprint, Image Foresting Transform, Optimum-Path Forest, Thermal Face Recognition.

\section{Introduction}

Biometric identification systems, which are based on physical, behavioral and physiological features of a given person, have been widely used in the last years as an alternative to increase the security level or replace the traditional identification systems, which are mainly based on possession or knowledge.

Although fingerprint recognition still remains the most used biometric technique, such approach is very sensitive to fingers' imperfections, which can be congenital or acquired over time. Other alternatives have been extensively pursued, such as iris and face recognition in visible spectrum. While the former has prohibitive costs, the latter is extremely dependent on the environment illumination and is not straightforward to distinguish twins or similar people.

Therefore, aiming to get deeper with face recognition, thermal face imagery has been used, since the temperature in the different regions of the human face allow its characterization. This thermal map is directly related to the blood vessels network of the face, which is unique for each individual [5].

Keeping up this in mind, several works have proposed new approaches for face recognition in infrared spectrum using physiological features. Akhloufi and Bendada [1], for instance, introduced the concept of faceprint, in which the

\footnotetext{
* Supported by Fapesp Grant \# 2009/12437-9.

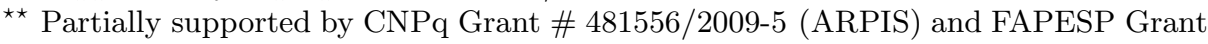
\# 2009/16206-1.
} 
physiological features of the face are extracted by identifying the boundaries of isothermal regions. The final result looks like a traditional fingerprint, with the crests representing the vessels and the valleys the isothermal regions.

Buddharaju et al. [5] provided a comprehensive study on physiological-based face recognition. Their work proposed a methodology to segment the blood vessels network and extract the thermal minutia points in order to compare the faces of a given database. Later, Buddharaju and Pavlidis [3] proposed a new methodology to correct the problems of false acceptance ratio in their previous work, mainly because of the methodological weakness in the feature extraction and face matching algorithms. Thus, the main contributions of their work were twofold: (i) the first one is related with the blood vessels segmentation step, which was improved in order to remove false contours of the vascular network, and (ii) the second one concerns with the development of a novel face matching algorithm, which considers pose deformations and face expression changes.

Chel et al. 6] evaluated the application of PCA technique in infrared images, and also reported its impact over images acquired with different conditions of illumination, expression and appearance. Finally, Buddharaju et al. [4] proposed a feature-based approach that characterizes the shape of isothermal regions, for further face recognition.

This paper proposes a framework to compute the faceprint using a set of graph-based tools provided by the Image Foresting Transform (IFT) [9] and by Optimum Path Forest (OPF) [11. While the former addresses image processing techniques, the latter handles unsupervised classification. To the best of our knowledge, this is the first time that IFT and OPF are used to this purpose. The remainder of this paper is organized as follows. Section 2 describes IFT and OPF. The proposed methodology is presented in Section 3. Finally, conclusions and future works are stated in Section 4.

\section{$2 \quad$ Background Theory}

In this section we introduce some basic concepts about the Image Foresting Transform and the Optimum-Path Forest.

\subsection{Image Foresting Transform}

The Image Foresting Transform proposed by Falcão et al. 9] is a graph-based tool to the design of image processing operators. Each pixel is modeled as a node, and a predefined adjacency relation originates a graph over the image. After that, the IFT algorithm begins a competition process between some key samples (seeds) in order to partition the graph into optimum-path trees (OPTs), which are rooted at the seeds.

The competition process is ruled by a path-cost function that needs to be constrained under some restrictions [9], and may simulate a region growing process in the graph. As one can see, by selecting a proper adjacency relation, seeds estimation methodology and path-cost function, one can design an image processing 
operator based on the IFT paradigm. Actually, the IFT can be seen as a generalization of the Dijkstra's algorithm to compute shortest paths, in the sense that IFT allows to use different path-cost functions and with multiple source nodes.

One can find several implementations of IFT-based segmentation. In this work, we used the IFT-WT (IFT-Watershed) approach 9], which works similar to the Watershed algorithm [12. More details about IFT can be found in [9].

\subsection{Optimum-Path Forest}

Keeping in mind the idea of IFT, the Optimum-Path Forest is a framework to the design of pattern classifiers based on discrete optimal partitions of the feature space. In this case, each dataset sample is represented by its corresponding feature vector in a node, which originates a graph together with a predefined adjacency relation. Both nodes and arcs can be weighted with density values and the distance between feature vectors, respectively.

Given some key samples (prototypes), which work similar to the seeds in the IFT algorithm, the OPF tries to partition the graph in OPTs, which may represent clusters (unsupervised classification) or labeled trees (supervised classification). In this work, we applied the unsupervised OPF, also known as OPF clustering, in order to group isothermal regions of the face.

In this case, the prototypes are chosen as the samples that fall in the regions with highest density. The OPF clustering uses a $k$-nn adjacency, in which the best value of $k$ is chosen as the one the minimizes a minimum cut over the graph [11, and it is bounded by $k_{\max }$. This parameter is chosen by user, and is responsible to allow a wider search range for OPF to compute the density of each node, which is calculated over its $k$-neighborhood.

\section{Graph-Based Framework}

Facial images obtained through infrared devices contain thermal information about the blood vessels network, which originates regions with different temperatures (Figure 3a). This thermal map produces an unique thermal faceprint for each individual [1].

In this work, we propose a novel framework to obtain faceprints from thermal images composed by four steps, as described by Figure 1. Each one of these steps is modeled to be conducted with the graph-based framework described in the previous section. These steps will be further detailed in the next sections. In order to validate the proposed method, we used images obtained from UND Collection $\mathrm{C}$ dataset [7].

\subsection{Pre-processing}

In infrared images, a set of possible face transformations (rotation, scaling and translation) and sensor-dependent variations (e.g., automatic gain control calibration and bad sensor points) could undermine the recognition performance. 


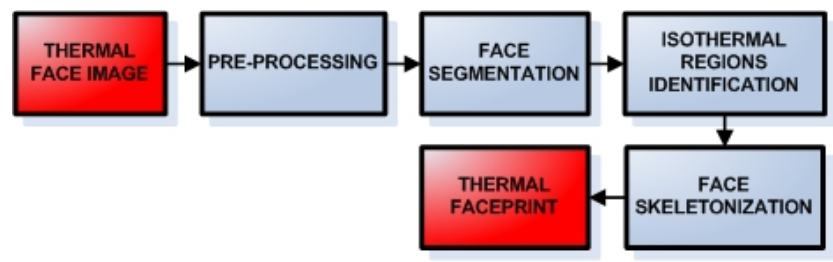

Fig. 1. General pipeline for the proposed methodology: the blue boxes denote the four main steps, while the red ones mean the input and output to the system

This impact can be minimized by performing some pre-processing operations, as follows:

1. Integer to float conversion to proceed with pixel-based operations at the image;

2. Pixel normalization to compensate brightness and contrast variations and

3. Histogram equalization in order to reduce image variation due to lighting and sensor differences.

\subsection{Face Segmentation}

The image segmentation concerns with to divide an image in regions that share certain features, aiming to separate the object of interest from its background. In our proposed approach, we divide the segmentation process in several steps in order to minimize errors in the face extraction procedure. These steps provide the basis for the proposed face extraction methodology. Figure 2 displays the detailed pipeline for the face segmentation schema adopted here.

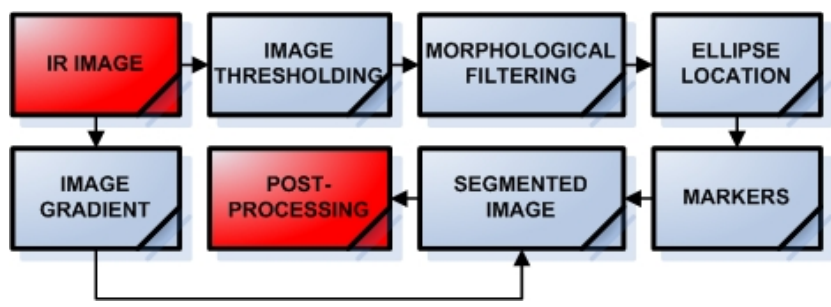

Fig. 2. Proposed face segmentation pipeline composed by five main steps: image thresholding, morphological filtering, ellipse location, markers finding and post-processing

Image thresholding. This first step attempts to separate the individual's face from its background. Given that thermal face images are characterized by high contrast between background and foreground, we applied the Otsu threshold [10] in order to obtain a binary image. Figures $3 \mathrm{a}$ and $3 \mathrm{~b}$ display the original and thresholded images, respectively. 
Morphological Filtering. Although the thresholding process may achieve good results, some images still could contain imperfections, such as face pixels disconnected from the foreground and holes inside the region of interest. In order to circumvent such problems, we performed morphological closing and opening operations at the images of the database, as shown in Figure 3 .

Ellipse Location. Given that a human face has quite similarity with the geometric figure defined by an ellipse, this step consists in finding the biggest ellipse contained at the image's background. Such task was accomplished by using an IFT-based method to find ellipses proposed by Andaló et al. [2]. The idea is to find the biggest ellipse within a homogeneous region with a center in a point that belongs to this region (Figure $3 \mathrm{~d}$ ).

Markers. The next step consists in to execute the segmentation process using the IFT-WT, which requires the use of both internal (foreground) and external (background) markers. In order to automatically find them, we propose here to use the ellipse found in the previous step as the basis to compute the internal and external markers. Since we have the ellipse (Section [3.2), we may execute erosion and dilation operations on that in order to find the internal and external ellipses (Figure 3e), that are used as internal and external markers, respectively. This eliminates the need of markers manually selected by user.

The segmentation is then performed using the markers at gradient image (Figure $3 \mathrm{f}$ ). The result of the segmentation is shown in Figure 3 s.

\subsection{Post-processing}

The last step consists in to post-process the segmented face, since some images still have some imperfections (Figure $3 \mathrm{~g}$ ). The idea is to obtain the binary mask of the segmented image and then run the ellipse location step again (Section (3.2). The final result is obtained by applying the ellipse's mask to the original image (Figure $3 \mathrm{a}$ ). Figure 3h displays the resulting face extracted after the post-processing procedure.

\subsection{Isothermal Regions Identification}

This step comprises with the identification of the isothermal regions, that is, regions that have homogeneous temperature, which are related to the pixels's brightness. We propose to handle the isothermal regions identification using the OPF clustering algorithm introduced by Rocha et al [11. For that, we used $k_{\max }=100$.

The value used for $k_{\max }$ was chosen after several experiments, and seemed to be the best option in our case. Low values of $k_{\max }$ lead us to an over clustering, whereas high values tend to merge regions. The reason for that relies on the fact that the best $k$ value is chosen as the one that minimizes a minimum cut over the graph [1], and its search range is bounded by $k_{\max }$. 


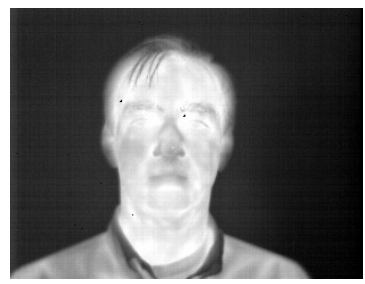

(a)

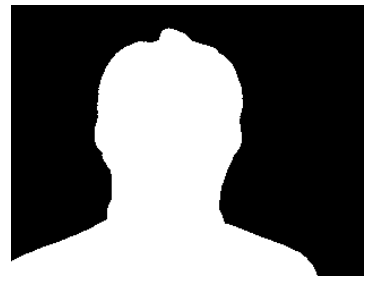

(c)

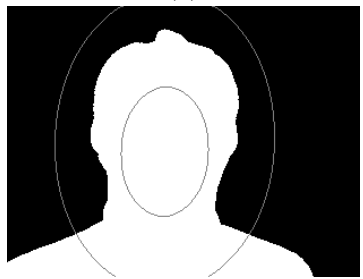

(e)

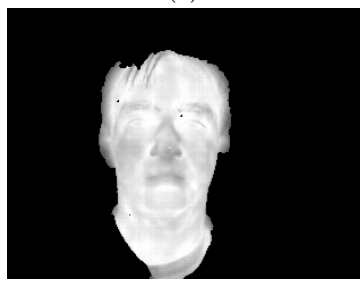

$(\mathrm{g})$

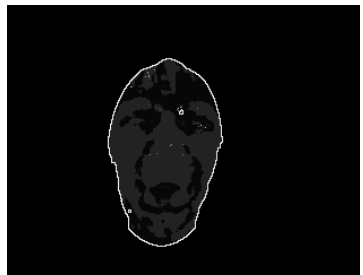

(i)

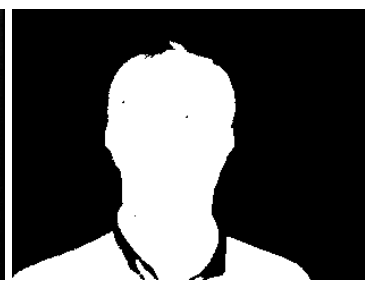

(b)

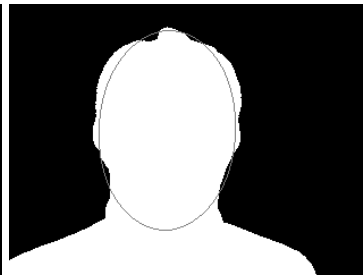

(d)

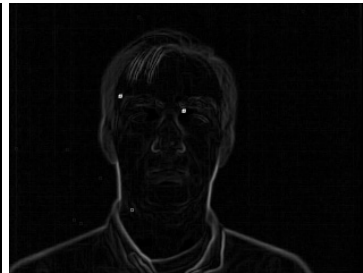

(f)

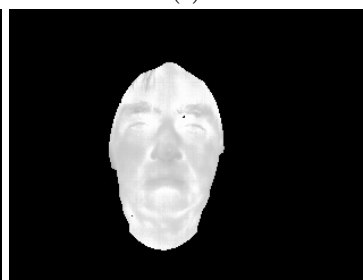

(h)

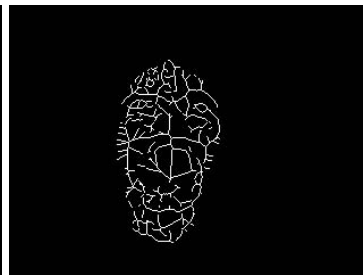

(j)

Fig. 3. Proposed methodology for faceprint extraction: (a) original thermal image in grayscale ( 8 bits/pixel), (b) thresholded image according to Section 3.2 (c) image after morphological filtering (Section 3.2), (d) ellipse location at face (Section 3.2), (e) internal and external markers defined by the eroded and dilated ellipses, respectively, (f) gradient image, (g) segmented image with some imperfections, (h) face extracted at the final of the segmentation process described in Figure 2 (i) face with isothermal regions grouped by OPF clustering and (j) faceprint obtained at the final of the proposed methodology depicted in Figure 1 


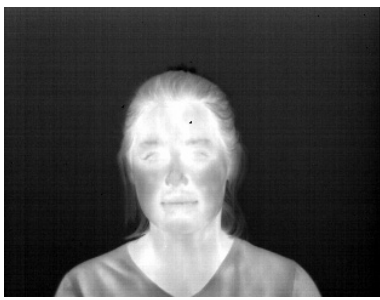

(a)

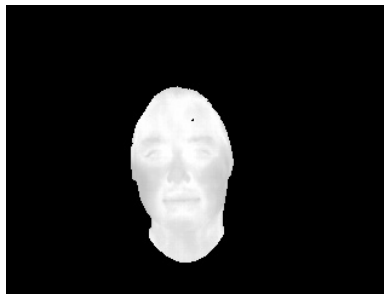

(c)

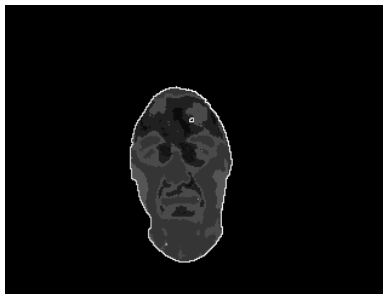

(e)

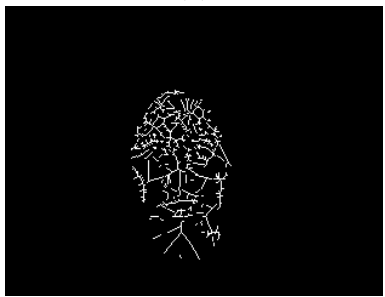

(g)

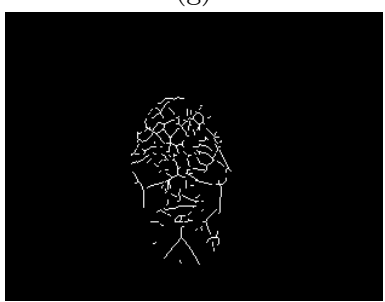

(i)

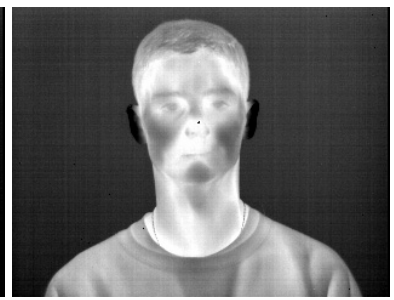

(b)

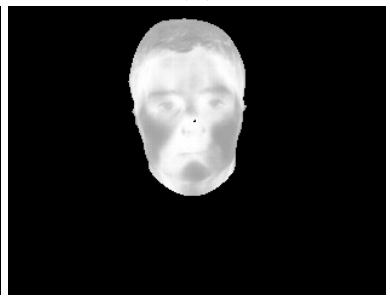

(d)

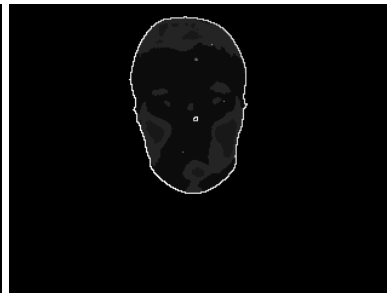

(f)

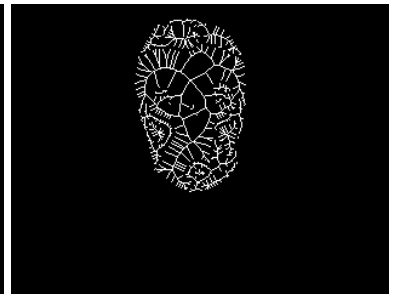

(h)

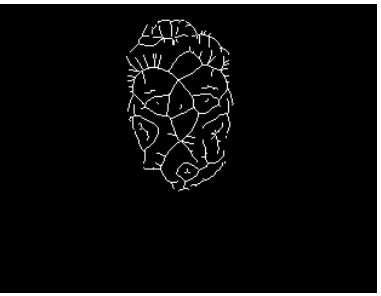

(j)

Fig. 4. Proposed methodology applied to two images of the UND Collection C dataset [7]: (a)-(b) infrared images, (c)-(d) face extracted according to Section 3.2. (e)-(f) isothermal regions identified (Section 3.4), and skeletons obtained with $\gamma=1$ in $(\mathrm{g})-(\mathrm{h})$ and $\gamma=3$ in $(\mathrm{i})-(\mathrm{j})$ 


\subsection{Face Skeletonization}

The last step consists in the characterization of the isothermal regions through the face skeletonization. The edge representation of the objects by their internal and external skeletons has been studied for several years, since them are compact representations and allow to rebuild the object. In this work, we carried out this phase with an IFT-based algorithm proposed by Falcão et al. [8].

The main idea is to use each border pixel as a seed, and then label it with a consecutive integer value. Further, IFT propagates the contour and the pixel's labels, using a path-cost function based on the Euclidean distance. This process outputs three images: (i) contour label map, (ii) pixel label map and (iii) an image filtered with the Euclidean Distance Transform. By filtering the contour label map image with a procedure described in [8], one can obtain its internal skeletons.

However, the main drawback of such image representation concerns with the irrelevant branches produced during the image skeletonization. Thus, one need to consider a threshold $\gamma$ in order to prune them. Figure 4 displays the methodology applied to different individuals of UND Collection $\mathrm{C}$ dataset. Figures $4 \mathrm{~b}$, 4h, 4 . and $4 \mathrm{j}$ show the thermal faceprint obtained with different values of pruning thresholds.

\section{Conclusions}

In this paper we deal with the problem of face characterization in infrared imagery. Since that images acquired in the visible spectrum can be affected by distortions in illumination, thermal images may appear to overcome such problems.

Some recent works have proposed to build a faceprint of individuals, instead of carrying out with holistic methods for thermal face recognition. The faceprint is based on the blood vessels network of the face, which is unique for each individual, even for twins. Thus, we propose here a novel graph-based framework to obtain such faceprints, composed by the IFT and OPF methods. To the best of our knowledge, this is the first time that both techniques are applied to tackle this problem. We also presented a robust procedure to extract faces from thermal images. Nowadays, our ongoing research has been guided to extract information about faceprints in order to associated them to the individuals of the dataset.

\section{References}

1. Akhloufi, M., Bendada, A.: Infrared face recognition using distance transforms. In: Proceedings of the World Academy of Science, Engineering and Technology, vol. 30, pp. 160-163 (2008)

2. Andaló, F.A., Miranda, P.A.V., Torres, R.d.S., Falcão, A.X.: Shape feature extraction and description based on tensor scale. Pattern Recognition 43(1), 26-36 (2010) 
3. Buddharaju, P., Pavlidis, I.T.: Physiological face recognition is coming of age. In: Proceedings of the Conference on Computer Vision and Pattern Recognition, pp. 128-135. IEEE Computer Society, Los Alamitos (2009)

4. Buddharaju, P., Pavlidis, I.T., Kakadiaris, I.A.: Face recognition in the thermal infrared spectrum. In: Proceedings of the Conference on Computer Vision and Pattern Recognition Workshop, vol. 8, pp. 167-191 (2004)

5. Buddharaju, P., Pavlidis, I.T., Tsiamyrtzis, P., Bazakos, M.: Physiology-based face recognition in the thermal infrared spectrum. IEEE Transactions on Pattern Analysis and Machine Intelligence 29(4), 613-626 (2007)

6. Chen, X., Flynn, P.J., Bowyer, K.W.: Ir and visible light face recognition. Computer Vision and Image Understanding 99(3), 332-358 (2005)

7. Chen, X., Flynn, P.J., Bowyer, K.W.: Ir and visible light face recognition. Computer Vision and Image Understanding 99(3), 332-358 (2005)

8. Falcão, A.X., da Costa, L.F., Cunha, B.S.: Multiscale skeletons by image foresting transform and its application to neuromorphometry. Pattern Recognition 35(7), 1571-1582 (2002)

9. Falcão, A.X., Stolfi, J., Lotufo, R.A.: The image foresting transform: Theory, algorithms, and applications. IEEE Transactions on Pattern Analysis and Machine Intelligence 26(1), 19-29 (2004)

10. Otsu, N.: A threshold selection method from gray-level histograms. IEEE Transactions on Systems, Man and Cybernetics 9(1), 62-66 (1979)

11. Rocha, L.M., Cappabianco, F.A.M., Falcão, A.X.: Data clustering as an optimumpath forest problem with applications in image analysis. International Journal of Imaging Systems and Technology 19(2), 50-68 (2009)

12. Vincent, L., Soille, P.: Watersheds in digital spaces: An efficient algorithm based on immersion simulations. IEEE Transactions on Pattern Analysis and Machine Intelligence 13(6), 583-598 (1991) 